\title{
Exact Solution of a Drop-push Model for Percolation
}

\author{
Satya N. Majumdar and David S. Dean \\ Laboratoire de Physique Quantique (UMR C5626 du CNRS), Université Paul Sabatier, 31062 Toulouse Cedex, France.
}

(November 3, 2018)

\begin{abstract}
Motivated by a computer science algorithm known as 'linear probing with hashing' we study a new type of percolation model whose basic features include a sequential 'dropping' of particles on a substrate followed by their transport via a 'pushing' mechanism. Our exact solution in one dimension shows that, unlike the ordinary random percolation model, the drop-push model has nontrivial spatial correlations generated by the dynamics itself. The critical exponents in the droppush model are also different from that of the ordinary percolation. The relevance of our results to computer science is pointed out.
\end{abstract}

PACS numbers: 64.60.A, 02.50.-r, 05.40.-a, 89.20.-a

The ordinary site or bond percolation and its various generalizations are amongst the most well studied problems in statistical physics [1,2]. Motivated by a well known computer science algorithm known as 'linear probing with hashing' (LPH) [3], we introduce and study in this Letter a new type of percolation model. Borrowing a name from the models of activated flow through traps [1] we call this a 'drop-push' model since it has two basic features: a sequential 'dropping' of particles on a substrate followed by the transport of the dropped particles via a 'pushing' mechanism caused by the local hardcore repulsion between particles on the substrate. Unlike in the ordinary percolation, we show that the dynamics of the drop-push model generates nontrivial spatial correlations between sites. Our exact solution in one dimension shows that the critical exponents associated with the percolation transition in the drop-push model are different from those of the ordinary percolation. As an additional bonus, our approach also rederives, in a straightforward way, a key result on the cost function in the LPH algorithm obtained recently by computer scientists using more involved combinatorial techniques [5,66. Our model is also easily generalizable to higher dimensions.

The LPH algorithm was originally introduced by Knuth [3] and has remained popular in computer science due to its simplicity, efficiency and general applicability 57. It can be described as follows: Consider $M$ items $x_{1}$, $x_{2}, \ldots, x_{M}$ to be placed sequentially into a table with $L$ cells labelled $1,2, \ldots, L$. For each item $x_{i}$, a cell with label $h_{i} \in\{1,2, \ldots, L\}$ is selected. The label $h_{i}$ is called the hash address and is usually chosen randomly from the set $\{1,2, \ldots, L\}$. The item $x_{i}$ is inserted at the $h_{i}$-th cell provided it is empty. Otherwise one tries cells $h_{i}+1$, $h_{i}+2$, etc. until an empty cell is found (the locations of the cells are interpreted modulo $L$ ) where the item $x_{i}$ is finally inserted. From the computer science point of view, the object of interest is the cost function $S(M, L)$ defined as the total number of unsuccessful probes encountered in inserting the $M$ items into a table of size $L$ [5.6]. This cost function measures the construction cost of the table as well as the time to search for an item later
[5,6]. The statistics of this cost function were found to be very different in the sparse table (when the density $M / L<<1$ ) compared to the full table (when $M / L \rightarrow 1$ ) [5]. We show below that, when interpreted as an interacting particle system, this crossover from the sparse to full table corresponds precisely to a percolation transition which belongs to a different universality class than that of the ordinary site or bond percolation.

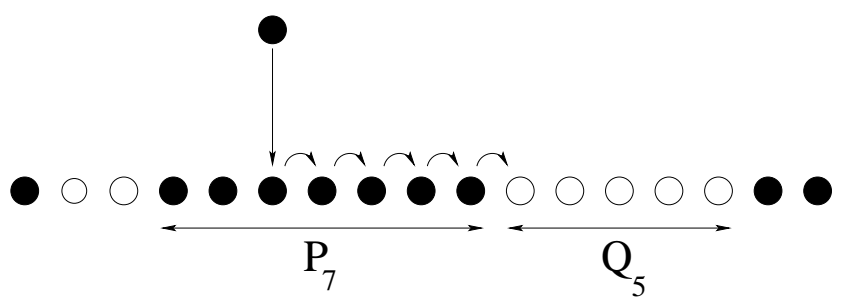

FIG. 1. The dropping and the subsequent hopping moves in the drop-push model. Also shown a particle cluster of size 7 and a hole cluster of size 5 .

In our equivalent drop-push model (Fig. 1), we interpret the table as a lattice of size $L$ with periodic boundary conditions. The cells are the lattice sites and each site can contain at most one particle. One starts with an empty lattice. At each step a particle is dropped into a randomly selected site. If the target site is empty, the incoming particle occupies it. If however the site was already occupied, the particle keeps hopping to the right until it finds an empty site where it then stays (see Fig. 1). One then repeats the same procedure with the next particle and so on. The dropping process in this model is similar to that of random sequential adsorption (RSA) and the car parking processes [7], however the adsorption mechanism in the drop-push model is quite different from the usual RSA models. In the car parking language, in this drop-push model, a new car arrives at a random spot on a one way lane and moves forward till it finds an empty parking spot. Unlike usual car parking models, here a car always manages to find a spot and thus the system never gets stuck in a jammed state. Note that although we have defined the hopping of the particle to be 
unidirectional, one can also consider an unbiased version where a particle, if dropped onto an occupied cluster, performs an unbiased random walk on the occupied cluster till it finds an empty site where it is then attached. We show below that in one dimension, the results are independent of this bias.

Each addition of a new particle corresponds to incrementing the density $t=M / L$ by the amount $\Delta t=1 / L$. Thus in the thermodynamic limit $L \rightarrow \infty$, the density $t$ becomes a continuous 'time' like variable that increases monotonically from $t=0$ (empty lattice) to $t=1$ (full lattice). For convenience, we will henceforth refer to the density $t$ as 'time' with $0 \leq t \leq 1$. The central objects of our analysis are $P_{n}(t)$ and $Q_{n}(t)$ denoting respectively the number (per unit length) of particle and hole clusters of size $n$ at time $t$ (see Fig. 1). The total number of particle (hole) clusters is denoted by $N(t)=\sum_{n} P_{n}(t)=\sum_{n} Q_{n}(t)$. Note that as $t$ increases continuously from 0 to 1 , one expects that $N(t)$ starting from $N(0)=0$ should increase initially but will eventually decrease to 0 again as $t \rightarrow 1$ when the lattice is nearly full. Thus $N(t)$ has an interesting nonmonotonic behavior in $0 \leq t \leq 1$ with a maximum (when the system has the largest number of clusters) at an intermediate time $t^{*}$ (see the inset of Fig. 2). We also note the sum rules corresponding to the particle and the hole densities: $\sum_{n} n P_{n}(t)=t$ and $\sum_{n} n Q_{n}(t)=(1-t)$.

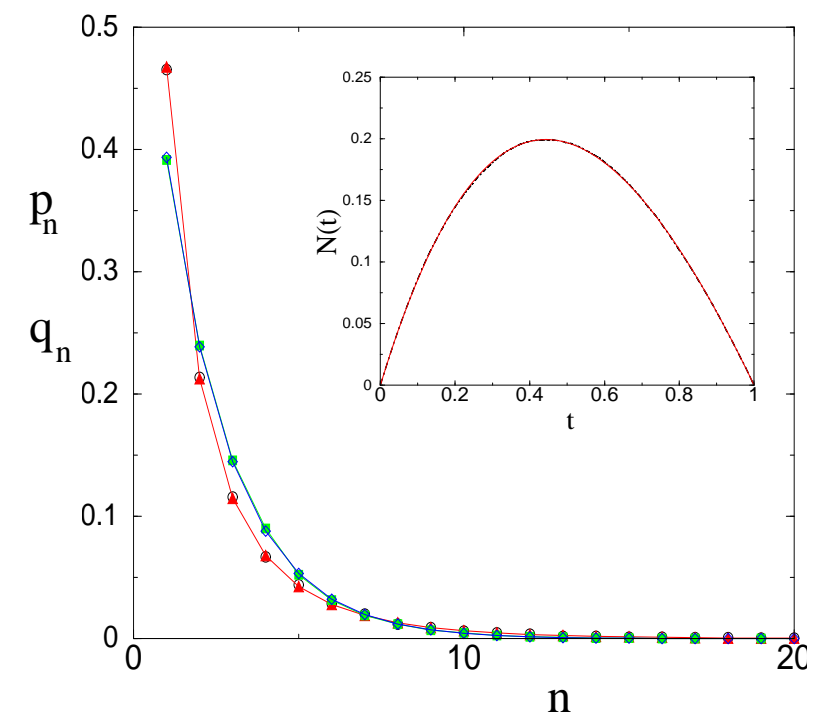

FIG. 2. The exact solutions for the normalized cluster densities $p_{n}=P_{n} / N$ (filled triangles) and $q_{n}=Q_{n} / N$ (filled squares) are compared with numerical results (circles and diamonds respectively) obtained via the Monte Carlo simulation of the drop-push model on a lattice with $L=100000$ at the filling factor $t=0.5$. The inset shows a plot of the numerical domain density $N(t)$ and the exact result $N(t)=(1-t)\left(1-e^{-t}\right)$. The two curves are indistinguishable.
The key observation that allows the exact solution in one dimension is the fact that the 'cluster' or 'domain' densities $P_{n}$ 's and $Q_{n}$ 's are statistically independent at all times $0 \leq t \leq 1$. This follows from the fact that the adsorption of a new particle at the edge of a particle (hole) cluster does not introduce correlations between the adjacent particle and hole clusters. A rigorous justification of this fact, details of which will be published elsewhere [8], follows from the observation that the domain walls in the drop-push model can be viewed as the zero crossings of a Markov process in space (at a fixed time $t$ ). Thus if one labels a configuration $\mathcal{C}$ by the set $\left\{n_{i}\right\}$ where $n_{i}$ 's denote the lengths of the alternate particle and hole clusters, then the probability of $\mathcal{C}$ is given by the product measure, $\operatorname{Prob}[\mathcal{C}, t] \propto P_{n_{1}}(t) Q_{n_{2}}(t) P_{n_{3}}(t) Q_{n_{4}}(t) \ldots$.. In other words, the independent interval approximation (IIA) is exact in this model. The next step is to write down the exact rate equation of evolution of $P_{n}$ 's and $Q_{n}$ 's by accounting for all the gain and loss terms due to the addition of a new particle and exploiting the factorization property of the probabilities. Similar types of IIA equations have been used in one dimensional coarsening problems [9]. The rate equation for the $Q_{n}$ 's turn out to be simple

$$
\frac{d Q_{n}}{d t}=-\left(n+\frac{t}{N}\right) Q_{n}+2 \sum_{m=n+1}^{\infty} Q_{m}+\frac{t}{N} Q_{n+1},
$$

valid for all $n \geq 1$. The first term denotes the loss of a hole cluster of size $n$ due to an adsorption of a particle by direct hit at any of the available $n$ sites and also due to a hit at any occupied site of the neighboring particle cluster to the left which then transports the particle to the leftmost site of the hole cluster. The latter happens with rate $\sum_{k} k P_{k} / N=t / N$. The second term denotes a gain in $Q_{n}$ due to a direct hit inside a hole cluster of size bigger than $n$. The factor 2 denotes that there are only two places available for the incoming particle in order to generate a hole cluster of size $n$ from a bigger hole cluster. The third term indicates a gain of a hole cluster of size $n$ from that of size $n+1$ due to a particle adsorption at its left edge. One can similarly write down the equations for the $P_{n}$ 's though they turn out to be trickier. Omitting the details [8], we present only the final results

$$
\begin{aligned}
\frac{d P_{n}}{d t} & =-\left(n+2+\frac{t Q_{1}}{N^{2}}\right) P_{n}+\left(1-\frac{Q_{1}}{N}\right)(n+1) P_{n-1} \\
& +\frac{Q_{1}}{N^{2}} \sum_{j=1}^{n-2}(j+1) P_{j} P_{n-1-j}, \quad n \geq 2 \\
\frac{d P_{1}}{d t} & =-\left(3+\frac{t Q_{1}}{N^{2}}\right) P_{1}+\sum_{m=2}^{\infty}(m-2) Q_{m} .
\end{aligned}
$$

Although the above equations are written down for the unidirectional version of the model, a careful analysis shows that they remain unchanged for the general case 
in which the dropped particle moves to the right with probability $p$ or to the left with probability $(1-p)$ [ 8 .

As an important consistency check, one can verify that both the Eqs. (11) and (2) satisfy the respective sum rules $\sum_{n} n Q_{n}=(1-t)$ and $\sum_{n} n P_{n}=t$. One can also write down the evolution equation for the total domain density via direct inspection of the process,

$$
\frac{d N}{d t}=-2 N-\frac{t}{N} Q_{1}+1-t .
$$

It is easy to check that both Eqs. (11) and (2) when summed over $n$ satisfy Eq. (3) thus providing yet another useful consistency check.

We note that Eq. (1) for the $Q_{n}$ 's does not involve the $P_{n}$ 's, but is however implicitly nonlinear due to the occurrence of $N=\sum_{n} Q_{n}$ on the right hand side. However Eq. (1) admits a very simple pure exponential solution as found in many RSA models [7]. The ansatz $Q_{n}(t)=A(t) \exp [-n B(t)]$ satisfies Eq. (11) for all $n \geq 1$ with the choice $A(t)=2(1-t)[\cosh (t)-1]$ and $B(t)=t$. One then gets the exact solution for the domain density $N(t)=\sum_{n} Q_{n}(t)=(1-t)\left(1-e^{-t}\right)$ which is nonmonotonic in $0 \leq t \leq 1$ and is asymmetric about its unique maximum at $t^{*}=0.4428 \ldots$ (see Fig. 2). Note that in the ordinary site percolation in 1-d with occupation probability $t$, the domain density is simply given by $N(t)=t(1-t)$ which is symmetric about the maximum at $t^{*}=1 / 2$.

We next substitute the exact solution for the $Q_{n}$ 's into Eq. (2) and first solve for $n=1$ and $n=2$. The exact solutions $P_{1}(t)=t(1-t) e^{-2 t}$ and $P_{2}(t)=3 t^{2}(1-t) e^{-3 t} / 2$ suggest the ansatz: $P_{n}(t)=a_{n}(1-t) t^{n} e^{-(n+1) t}$. Indeed this ansatz solves Eq. (2) provided the $a_{n}$ 's satisfy the nonlinear recursion relation,

$$
n a_{n}=(n+1) a_{n-1}+\sum_{i=1}^{n-2}(i+1) a_{i} a_{n-1-i}
$$

starting with $a_{1}=1$. The first few values are $a_{2}=3 / 2$, $a_{3}=8 / 3, a_{4}=125 / 24$ etc. From Eq. (4) it follows that the generating function $\tilde{a}(z)=\sum_{n=1}^{\infty} a_{n} z^{n}$ is given by $\tilde{a}(z)=-1+T(z) / z$ where $T(z)$ is the well known tree function given by the solution of the equation $T(z) \exp [-T(z)]=z$. It is easy to see that the function $T(z)$ has a singularity at $z=1 / e$ where $T(z) \approx 1-\sqrt{2(1-e z)}+\ldots$ This tree function appears in problems related to the counting of rooted labelled trees [10] with various applications in computer science [3,5] as well as many physics problems such as aggregation models 11] and the classical hard sphere fluid in large dimensions [12]. Using the known properties of $T(z)$, we then get $a_{n}=(n+1)^{n-1} / n$ ! for all $n \geq 1$. We have also checked that the exact solutions

$$
\begin{aligned}
& Q_{n}(t)=(1-t)\left(e^{t}-1\right)^{2} e^{-(n+1) t} \\
& P_{n}(t)=(1-t) t^{n} e^{-(n+1) t} \frac{(n+1)^{n-1}}{n !}
\end{aligned}
$$

match perfectly with the numerical results obtained via the Monte Carlo simulation of the drop-push model (see Fig. 2).

Clearly at $t=1$, there is only one infinite particle cluster and the system percolates. We now analyze the scaling behavior of the cluster distributions near the critical point $t=1$. From Eq. (6), we find that for large $n, P_{n}(t) \approx \frac{(1-t)}{\sqrt{2 \pi}} n^{-\theta} \exp \left[-n / n^{*}(t)\right]$ where $n^{*}(t)=1 /(t-1-\log t)$ and the Parisi-Sourlas exponent 113 is given exactly by $\theta=3 / 2$. Note that for the ordinary percolation in one dimension, $P_{n}(t)=(1-t)^{2} t^{n}$ indicating $\theta=0$. In the limit $t \rightarrow 1$, the typical cluster size diverges as $n^{*}(t) \approx 2(1-t)^{-2}$ and one obtains the Stauffer scaling form [1] for the cluster size, $P_{n}(t) \sim n^{-\tau} f\left[n(1-t)^{\sigma}\right]$ with the Fisher exponent $\tau=2$ (as in the ordinary percolation) and $\sigma=2$ (in contrast to the ordinary percolation where $\sigma=1$ ). The exact scaling function here $f(z)=\sqrt{\frac{z}{2 \pi}} e^{-z / 2}$ also differs from that of the ordinary percolation where $f(z)=z^{2} e^{-z}$ [1]. Consequently the susceptibility exponent [1] given by the scaling relation $\gamma=(3-\tau) / \sigma$ also differs in the two models. For the drop-push model $\gamma=1 / 2$, where as $\gamma=1$ for the ordinary percolation.

We now turn to the correlation function $G_{n}(t)$ denoting the probability that two occupied sites separated by a distance $n$ belong to the same cluster at time $t$. For convenience we introduce the binary variable $\sigma_{i}$ such that $\sigma_{i}=1$ if the site $i$ is occupied and $\sigma_{i}=0$ otherwise. Then by definition $G_{n}=\left\langle\sigma_{i} \sigma_{i+1} \ldots \sigma_{i+n}\right\rangle$. We also note that by definition the particle cluster density $P_{n}=\left\langle\bar{\sigma}_{i} \sigma_{i+1} \ldots \sigma_{i+n} \bar{\sigma}_{i+n+1}\right\rangle$ where $\bar{\sigma}_{i}=1-\sigma_{i}$. As a consequence one obtains the exact relation, $G_{n+1}+$ $G_{n-1}-2 G_{n}=P_{n}$. Using the exact scaling form of $P_{n}$ and integrating twice with respect to $n$ we find that in the scaling limit $t \rightarrow 1, n \rightarrow \infty, G_{n}(t) \approx g\left[n(1-t)^{\nu}\right]$ where the correlation length exponent $\nu=2$ and the exact scaling function $g(z)=(1+z) \operatorname{erfc}\left(\sqrt{\frac{z}{2}}\right)-\sqrt{\frac{2 z}{\pi}} e^{-z / 2}$. These results should be compared to those for the ordinary percolation with occupation density $t$ where $G_{n}(t)=t^{n}$ indicating that $\nu=1$ and $g(z)=e^{-z}$ trivially.

To elucidate the nontrivial spatial correlations in the drop-push model we have also computed the conventional two point correlation function, $C_{n}(t)=\left\langle\sigma_{i} \sigma_{i+n}\right\rangle-$ $\left\langle\sigma_{i}\right\rangle\left\langle\sigma_{i+n}\right\rangle$, the connected part of the joint probability that two sites at distance $n$ are both occupied. Note that for the ordinary percolation, $C_{n}(t)=0$ for all $n \geq 1$ and $0 \leq t \leq 1$. In contrast, we show that $C_{n}(t)$ is nontrivial in the drop-push model. In order to compute it, we add up the possibilities that there may be no holes between the two sites, or maybe only one hole cluster, or two hole clusters etc. This method of expressing the correlation function in terms of the interval size distributions was used before in other contexts 14,15. Omitting details 
we present only the final expression for the generating function $\tilde{C}(z)=\sum_{n} C_{n}(t) z^{n}$

$$
\tilde{C}(z)=\frac{1-t}{1-z}\left[z t-1+\frac{t(1-z)}{t-T\left(z t e^{-t}\right)}\right],
$$

where $T(z)$ is the tree function defined earlier. Using the properties of the tree function it is straightforward, though somewhat tedious [8], to derive the asymptotic scaling properties of $C_{n}(t)$. We find after some algebra that in the scaling limit $t \rightarrow 1, n \rightarrow \infty$ but keeping $z=n(1-t)^{2}$ fixed, $C_{n}(t) \approx(1-t)^{2} F\left[n(1-t)^{2}\right]$ where the exact scaling function $F(z)=\frac{1}{\sqrt{2 \pi z}} e^{-z / 2}-\frac{1}{2} \operatorname{erfc}\left(\sqrt{\frac{z}{2}}\right)$. The function $F(z) \approx 1 / \sqrt{2 \pi z}$ as $z \rightarrow 0$ and $F(z) \approx$ $\frac{1}{\sqrt{2 \pi} z^{3 / 2}} e^{-z / 2}$ as $z \rightarrow \infty$.

We now turn to the total cost function $S(t)$ in the LPH algorithm, i.e. in the drop-push model with unidirectional transport. The cost $\Delta S(t)$ to insert a new particle at time $t$ is precisely the expected number of unsuccessful probes, i.e., the expected number of hops that the new particle undergoes before getting adsorbed into the right edge of a particle cluster. Consider a particle cluster of size $k$ at time $t$. The incoming particle can drop anywhere on this cluster and the number of hops is simply the distance of the target site from the right edge of the cluster. Noting that the cluster size can vary from 1 to infinity, one then gets $\Delta S(t)=\sum_{k=1}^{\infty}\left(\sum_{j=1}^{k} j\right) P_{k}(t)=\frac{1}{2} \sum_{k=1}^{\infty} k(k+1) P_{k}(t)$. Using the exact result for $P_{k}(t)$ from Eq. (6) we get $\Delta S(t)=t(2-t) /\left[2(1-t)^{2}\right]$. The total cost function is then given by $S(t)=\int_{0}^{t} \Delta S\left(t^{\prime}\right) d t^{\prime}=t^{2} /[2(1-t)]$, in agreement with the result derived by the computer scientists using rather involved combinatorial techniques [5.6. More details on the statistics of this cost function for finite size tables have also been derived recently [5:6].

Finally this drop-push model can be easily generalized to higher dimensions. Let us consider, for simplicity, the the unbiased version. One starts with an empty lattice of linear size $L$ in $d$-dimensions with periodic boundary conditions. At each step one drops a particle at a randomly chosen site. The incoming particle occupies the target site provided it was empty. Otherwise the dropped particle performs a random walk starting at the target site until it finds an empty site and sticks there. One then adds another particle and the process continues. It follows from the simple electrostatic analogy that when a particle drops onto an occupied cluster, it has equal probability to subsequently stick to any of the surface sites of this cluster. In this sense this model to similar to the celebrated Eden model 16]. However, unlike the Eden model, here one can have many different seed sites from which a new cluster can grow. Also the probability that a given cluster will grow by absorbing a new particle is proportional to the volume of the cluster in the drop-push model. Note that this model is also different from the previously studied cooperative adsorption models [17, the cluster-cluster aggregation models [16] and the random dynamical percolation model 18. It is clear that there will be a critical density $t=t_{c}<1$ at which the particle clusters start to percolate. An outstanding question is whether this percolation transition in higher dimensions, as in the 1-d case, belongs to a different universality class than that of the ordinary site percolation. Our preliminary numerical simulations in 2 -d indicate that indeed this may be the case [8]. We defer these results and other details for a future communication.

We thank M. Barma and D. Dhar for useful discussions.

[1] D. Stauffer and A. Aharony, Introduction to Percolation Theory, 2nd ed. (Taylor and Francis, London, 1992).

[2] For various generalizations of the ordinary percolation see the articles in the special issue, Physica A 266 (1999).

[3] D.E. Knuth, The Art of Computer Programming, 2nd ed. Vol-3 (Addison-Wesley, Reading, 1998).

[4] M. Barma and R. Ramaswamy in Nonlinearity and Breakdown in Soft Condensed Matter, edited by K.K. Bardhan, B.K. Chakrabarti, and A. Hansen (Springer, Berlin, 1993).

[5] P. Flajolet, P. V. Poblete, and A. Viola, Algorithmica, 22, 490 (1998).

[6] S. Janson, Random Struct. Alg. 19, 438 (2001).

[7] M.C. Bartelt and V. Privman, Int. J. Mod. Phys. B5, 2883 (1991); J.W. Evans, Rev. Mod. Phys. 65, 1281 (1993); J. Talbot, G. Tarjus, P.R. Van Tassel, and P. Viot, Colloid Surface A 165, 287 (2000).

[8] S.N. Majumdar and D.S. Dean, unpublished.

[9] P.L. Krapivsky and E. Ben-Naim, Phys. Rev. E 56, 3788 (1997); S.N. Majumdar, D.S. Dean, and P. Grassberger, Phys. Rev. Lett. 86, 2301 (2001).

[10] I. P. Goulden and D.M. Jackson, Combinatorial Enumeration (John Wiley, New York, 1983).

[11] P.G.J. van Dongen and M.H. Ernst, J. Phys. A: Math. Gen. 16, L327 (1983); J.L. Spouge, J. Phys. A: Math. Gen. 16, 767 (1983).

[12] H.L. Frisch, N. Rivier, and D. Wyler, Phys. Rev. Lett. 54, 2061 (1985).

[13] G. Parisi and N. Sourlas, Phys. Rev. Lett. 46, 871 (1981).

[14] P.A. Alemany and D. ben-Avraham, Phys. Lett. A 206, 18 (1995).

[15] S.N. Majumdar, C. Sire, A.J. Bray, and S.J. Cornell, Phys. Rev. Lett. 77, 2867 (1996); B. Derrida, V. Hakim, and R. Zeitak, ibid. 77, 2871 (1996).

[16] For a review of cluster growth models see L.M. Sander in Solids Far from Equilibrium, edited by C. Godrèche (Cambridge University Press, Cambridge 1992).

[17] D.E. Sanders and J.W. Evans, Phys. Rev. A 38, 4186 (1988).

[18] J.E. de Freitas, L. dos Santos Lucena, and S. Roux, Physica A, 266, 81 (1999). 